\title{
EDITORIAL
}

\section{IN THE MARCH, 2009 ISSUE OF CLINICS}

\author{
Mauricio Rocha-e-Silva, Editor
}

doi: $10.1590 / \mathrm{S} 1807-59322009000300001$

In this number of Clinics we highlight an intriguing study by Narayanan et al., triggered by the tremendous expansion of the use of cellular phones. They exposed Wistar rats to frequent bursts of the vibratory stimulation (no ring tone) produced by cellular phones and found that such stimulus induces a deficit of spatial memory performance using the Morris water maze test. Both groups did exhibit the expected reduction in the time necessary to learn the test but cellular phone exposed rats had significantly higher latencies to reach the target quadrant. They conclude that the cellular phone vibratory stimulus affected the acquisition of learned responses pointing out to a poor spatial navigation of the phone-exposed animals.

Alonso et al. compared the dislocation of the center of gravity and postural balance in sedentary and recreational soccer players with and without anterior cruciate ligament reconstruction. They examined soccer players with anterior cruciate ligament reconstruction, soccer players with no anterior cruciate ligament injuries, and sedentary subjects. They found that postural balance was worse on the reconstructed side of the athletes compared to their contralateral side, to both sides of the uninjured athletes and to the sedentary group.

Okay et al. describe variation among PCR systems in detecting Toxoplasma gondii as has been extensively reported and associated with target genes, primer composition, amplification parameters, treatment during pregnancy, host genetic susceptibility and genotypes of different parasites according to geographical characteristics. Amniotic fluid samples $(\mathrm{n}=$ 467) from T. gondii IgM- and IgG-positive Brazilian pregnant women being treated for 1 to 6 weeks at the time of amniocentesis were examined and authors conclude that the use of PCR for the diagnosis of fetal Toxoplasma infections

Hospital das Clínicas, Faculdade de Medicina da Universidade de São Paulo - São Paulo/SP, Brazil.

mrsilva36@hcnet.usp.br in Brazil should be targeted to the B1 gene when only one gene can be amplified, preferably by nested amplification with primers B22/B23.

Vieira et al. analyzed hemodynamic changes in 59 patients with cardiac valvar diseases submitted to dental treatment under local anesthesia containing epinephrine. They conclude that Lidocaine with epinephrine $(1: 100,000)$ provided effective local anesthesia and that this treatment did not increase heart rate or blood pressure, and did not cause any arrhythmic changes in patients with cardiac valvar diseases.

Camanho evaluated the clinical course of nine patients with ankylosis in their knees submitted to a total arthroplasty to lessen pain and improve functional limitation. For these patients, arthrodesis remained a possibility in the event of arthroplasty failure. He concludes that all of the patients experienced a significant reduction in pain and some improvement in the degree of knee flexion and extension.

Mentes et al. studied the benefits of ultrasonography for detecting the borders of pilonidal sinus tissue, using the correlation between physical and ultrasonographic examination for the surgical planning of 73 patients. They conclude that palpation and methylene blue injection do not provide appropriate information in many patients, whereas pre-operative ultrasonography can improve the identification of the sinus tract and its branches.

Sugaya et al. evaluated the efficacy of the drugs thalidomide, dapsone, colchicine, and pentoxifylline for treatment in 21 patients with recurrent aphthous stomatitis. The most efficient and best-tolerated drug was thalidomide. However, patients experienced relapses of the lesions after the withdrawal of their medication.

Carvalho et al. investigated the prevalence of pain complaints among 318 individuals with pathological knee 
conditions and evaluated palpation of the inferior pole of the patella as a diagnostic test for patellar tendinopathy. They found that 124 (39\%) individuals felt pain on palpation of the inferior pole of the patella. Of these, only 40 had received a diagnosis of patellar tendinopathy. They conclude that palpation of the inferior pole of the patella is a diagnostic procedure with high sensitivity and moderate specificity for diagnosing patellar tendinopathy, especially among individuals who perform activities with high functional demands.

Campos et al. developed a prosthesis for open pleurostomy cases where pulmonary decortication is not indicated, or where post-pneumonectomy space infection occurs. The prosthesis is a self-retained silicone tube that requires the removal of $3 \mathrm{~cm}$ of one rib for insertion, and acts as a mature conventional open pleural window. Forty-four consecutive patients were treated with this device in the management of different kinds of pleural empyema. They conclude that the prosthesis is a minimally invasive procedure that can be as effective as conventional open pleural window for management of chronic empyemas. Thus, they propose that the use of prosthesis for open pleurostomy should replace the conventional method.

Soares-Filho et al. determined the prevalence of anxiety and depression in patients complaining of chest pain who seek a chest pain unit attendance, and found that 59 of 130 patients presented Chest Pain of Determined Cause, and 71 of Indefinite Cause. They conclude that the high anxiety and depression prevalence observed may indicate the need for early and specialized approach to these disorders. When coronary arterial disease is present, this may decrease complications and shorten hospital stay. When psychiatric disorder appears isolated, it is possible to reduce unnecessary repeated visits to emergency room and increase patient's quality of life.

Barbosa et al. tested the hypothesis that changes in propofol PK increase the hypnotic effects after Cardiopulmonary bypass on 20 patients scheduled for on-pump or off-pump coronary artery bypass graft and found that de- spite similar plasma propofol concentrations in both groups, bispectral index values were lower in the on-pump coronary artery bypass graft group. They conclude that aside from the increased sensitivity of the brain to anesthetics after cardiopulmonary bypass, changes in propofol pharmacokinetics may contribute to its central nervous system effects.

Araujo et al. endeavored to identify relationships between sleep apnea, myocardial ischemia and cardiac arrhythmia in patients with coronary artery disease and conclude that the degree of obstructive sleep apnea may not increase myocardial ischemia and arrhythmias in patients with stable coronary artery disease.

Das et al. studied the effects of palm oil tocotrienol rich fraction on streptozotocin-induced diabetic rats and found that animals treated with the tocotrienol rich fraction exhibited intact vessel wall with reduced level of serum glucose concentrations, glycated haemoglobin, plasma total cholesterol, low-density lipoprotein cholesterol, triglyceride and higher level of high-density lipoprotein cholesterol.

Carnieto et al. endeavored to determine whether a selective cyclooxygenase-2 inhibitor (rofecoxib) could alter the evolution of acute myocardial infarction after reperfusion in a canine model (180 min of coronary occlusion, followed by 30 minutes of reperfusion). They note that Coronary flow and heart rate did not change in the treated animals, but that in the control group, there was blood pressure instability, in addition to maximal levels of CPK-MB post-infarction. The same results were observed for CPK-MBm and troponin I. They claim that selective inhibition of Cyclooxygenase-2 with rofecoxib was not associated with early detrimental effects on the hemodynamic profile or the gross extent of infarction, and that it may in fact be beneficial by limiting cell necrosis.

We also publish a review by Wichi et al. on the role of a chronic exercise intervention as a means to prevent autonomic nervous system changes during the aging process, and 3 case reports. 